\title{
Sliding Mode Disturbance Observer-Based Fractional Second-Order Nonsingular Terminal Sliding Mode Control for PMSM Position Regulation System
}

\author{
Hong-Ru Li, ${ }^{1}$ Zhi-Bin Jiang, ${ }^{1}$ and Nan Kang ${ }^{2}$ \\ ${ }^{1}$ School of Information Science and Engineering, Northeastern University, Shenyang 110819, China \\ ${ }^{2}$ Foreign Studies College, Northeastern University, Shenyang 110819, China \\ Correspondence should be addressed to Zhi-Bin Jiang; jiangzb1275031@126.com
}

Received 20 May 2014; Revised 14 August 2014; Accepted 18 August 2014

Academic Editor: Xing-Gang Yan

Copyright (C) 2015 Hong-Ru Li et al. This is an open access article distributed under the Creative Commons Attribution License, which permits unrestricted use, distribution, and reproduction in any medium, provided the original work is properly cited.

\begin{abstract}
This paper investigates the position regulation problem of permanent magnet synchronous motor (PMSM) subject to parameter uncertainties and external disturbances. A novel fractional second-order nonsingular terminal sliding mode control (F2NTSMC) is proposed and the finite time stability of the closed-loop system is ensured. A sliding mode disturbance observer (SMDO) is developed to estimate and make feedforward compensation for the lumped disturbances of the PMSM system. Moreover, the finitetime convergence of estimation errors can be guaranteed. The control scheme combining F2NTSMC and SMDO can not only improve performance of the closed-loop system and attenuate disturbances, but also reduce chattering effectively. Simulation results show that the proposed control method can obtain satisfactory position tracking performance and strong robustness.
\end{abstract}

\section{Introduction}

Permanent magnet synchronous motor has been widely applied in industrial automation, household appliances, computers, high-speed aerospace drives, and automobiles due to its superior properties such as high power density, high efficiency, low inertia, and reliable operation $[1,2]$. However, the PMSM system is a complex nonlinear system with multiple coupled states and unavoidable and unmeasured disturbances, as well as parameter perturbations. To achieve high-performance control, various advanced control methods have been proposed, such as adaptive control [3], robust control [4], sliding mode control [5, 6], optimal control [7], backstepping control [8], predictive control [9], fuzzy control [10], neural network control [11], finite-time control [12], fractional order control [13, 14], and intelligent control [15]. These methods have increased the dynamic and steady state performance of PMSM systems to some degree. Nevertheless, there still exist several obstacles as to complex control laws, conservative or excessive control gains [16], reliance on complete knowledge of the system model, and so forth.

Sliding mode control (SMC) is a well-known and efficient control technique to improve disturbance rejection and robustness of nonlinear systems and parameter estimation [17], and so forth. When system states are in the sliding mode, the closed-loop response becomes totally insensitive to both internal parameter uncertainties and external disturbances [18]. To further improve the transient performance of the closed loop system and ensure the finite-time convergence, terminal sliding mode control (TSMC) that employs nonlinear sliding surface is developed due to some superior properties such as faster, finite-time convergence and higher control precision [19]. The finite-time stabilization can bring dynamical systems to better robustness and disturbance rejection properties [16].

Zhankui and Sun [20] proposed a second-order fast terminal sliding mode control scheme that can not only 
guarantee tracking errors in approaching and reaching the sliding surface in finite time, but also improve tracking accuracy and eliminate the high frequency chattering of control inputs effectively. However, the second-order fast terminal sliding mode control still has a singularity problem. In Feng et al. [21] and $\mathrm{Yu}$ et al. [22], the nonsingular terminal sliding mode controllers (NTSMC) were designed to achieve finite-time tracking control of systems and overcome the singularity problem. Further, Yan et al. [23] combined NTSMC with second-order SMC to design the secondorder NTSMC (2NTSMC) for the finite-time convergence of system states. The 2NTSMC possess fast convergence and high control precision; besides, it can eliminate chattering behavior of control signals. In order to improve the control system robustness, the observer-based control method is often adopted, such as [24].

Fractional order calculus extends integer order to nonintegral order and provide an excellent tool for describing complex dynamic features. Recently, some researchers have proposed some fractional-order SMC methodologies. Dadras and Momeni [19] introduced fractional-order TSMC (FTSMC) to integer-order nonlinear systems. However, the chattering problem of control inputs still exists. Aghababa [25] designed a chatter-free terminal sliding mode controller for nonlinear fractional-order dynamical systems. However, to the authors' best knowledge, the order number of FTSMC is usually restricted to one and there is little work in which the order number of FTSMC is second or higher. Therefore, designing a FTSMC whose order number is greater than 1 for nonlinear dynamic systems is still an open problem.

To improve robustness during the reaching phase of SMC and reduce the conservativeness of selecting switching control gains, a sliding mode disturbance observer (SMDO) is employed to provide feed-forward compensation for parameter uncertainties and external disturbances. Consequently, the closed loop system can achieve global robustness and improve disturbance rejection performance.

In this paper, a new fractional second-order nonsingular terminal sliding mode controller (F2NTSMC) is proposed to ensure fast and finite-time convergence of the PMSM system. Then, a switching control law is determined to drive system states to the designed sliding surface and subsequently constrain system states to the surface hereafter. Meanwhile, the finite-time stability is proved by using fractional Lyapunov theory. Moreover, a SMDO is applied such that uncertainties and disturbance can be estimated and compensated. Eventually, simulation results verify good robustness and fast convergence of the proposed fractional control approach.

The rest of this paper is organized as follows. In Section 2, preliminaries of fractional-order calculus are introduced. In Section 3, the fractional-order PMSM system model and the problem formulation are presented. Section 4 copes with the proposed fractional-order approach and finitetime stability analysis. The effectiveness of the proposed control scheme is illustrated by numerical examples in Section 5. Finally, some concluding remarks are included in Section 6.

\section{Preliminaries of Fractional-Order Calculus}

Fractional-order integration and differentiation are the generalization of the integer-order ones [19]. Three commonlyused definitions for fractional order calculus are RiemannLiouville, Caputo, and Grünwald-Letnikov definitions as described below.

Definition 1 (see [26]). The $\alpha$ th-order fractional integration of function $f(t)$ with respect to $t$ and the terminal value $a$ are given by

$$
J_{a}^{\alpha} f(t)=\frac{1}{\Gamma(\alpha)} \int_{a}^{t}(t-\tau)^{\alpha-1} f(\tau) d \tau, \quad t \in[a, b], \alpha>0,
$$

and the $\alpha$ th-order Riemann-Liouville fractional derivative of function $f(t)$ is defined as

$$
\begin{aligned}
D_{a}^{\alpha} f(t)= & D^{n} J_{a}^{n-\alpha} f(t) \\
= & \frac{1}{\Gamma(n-\alpha)} \frac{d^{n}}{d t^{n}} \int_{a}^{t} \frac{f(\tau)}{(t-\tau)^{\alpha-n+1}} d \tau, \\
& a \leq t \leq b, \quad n=\lceil\alpha\rceil,
\end{aligned}
$$

where $f(t)$ is integrable on the closed interval $[a, b]$ and $\Gamma(\cdot)$ is Euler's gamma function.

Property 1 (see [26]). For the Riemann-Liouville derivative, we have

$$
\begin{array}{r}
D_{t_{0}}^{-\alpha}\left(D_{t_{0}}^{\beta} f(t)\right)=D_{t_{0}}^{\beta-\alpha} f(t) \\
-\sum_{j=1}^{m}\left[D_{t_{0}}^{\beta-j} f(t)\right]_{\mid t=t_{0}} \frac{\left(t-t_{0}\right)^{\alpha-j}}{\Gamma(1+\alpha-j)}, \\
m-1 \leq \beta \leq m .
\end{array}
$$

Definition 2 (see [26]). The Caputo fractional derivative of order $\alpha$ of a continuous function $f: R^{+} \rightarrow R$ is defined as follows:

$$
\begin{array}{r}
D_{* a}^{\alpha} f(t)=J_{a}^{n-\alpha} D^{n} f(t)=\frac{1}{\Gamma(n-\alpha)} \int_{a}^{t} \frac{f^{(n)}(\tau)}{(t-\tau)^{\alpha-n+1}} d \tau, \\
a \leq t \leq b, \quad n=\lceil\alpha\rceil .
\end{array}
$$

Definition 3 (see [26]). The Grünwald-Letnikov fractional derivative of order $\alpha$ of a continuous function $f: R^{+} \rightarrow R$ is defined as follows:

$$
{ }^{\mathrm{GL}} D_{a}^{\alpha} f(x)=\lim _{\substack{h \rightarrow \infty \\
m h=x-a}} \frac{1}{h^{\alpha}} \sum_{k=0}^{m}(-1)^{k}\left(\begin{array}{l}
\alpha \\
k
\end{array}\right) f(x-k h), \quad \alpha>0 .
$$

\section{Fractional-Order Model of PMSM}

Consider a typical PMSM vector control system, as shown in Figure 1. The differential equations of surface-mounted 


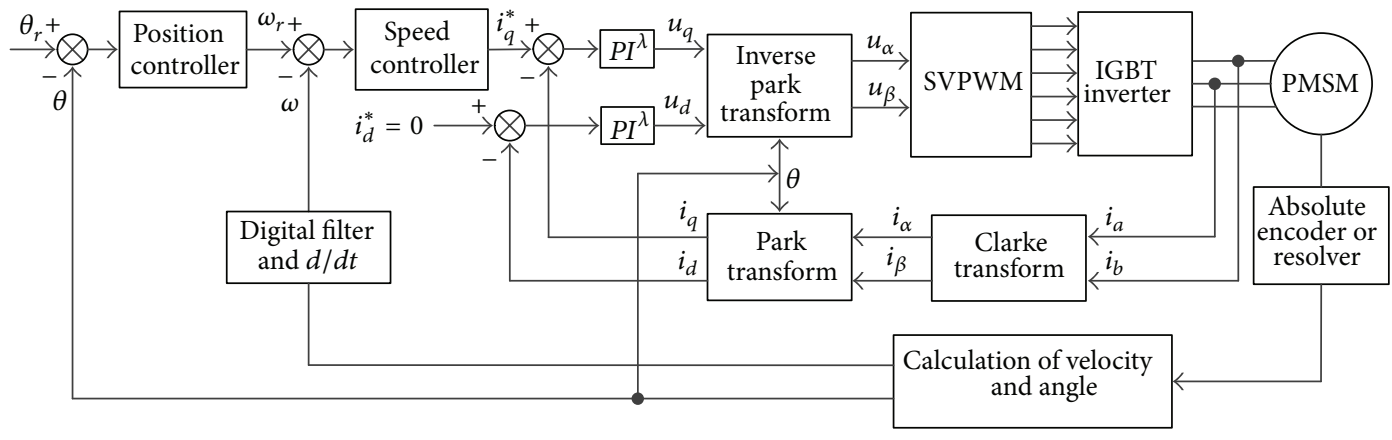

FIGURE 1: A typical PMSM system based on vector control.

PMSM represented in the rotor reference coordinates based on the assumptions [27] are given as follows:

$$
\left[\begin{array}{c}
\dot{i}_{d} \\
\dot{i}_{q} \\
\dot{\omega}_{r}
\end{array}\right]=\left[\begin{array}{ccc}
-\frac{R_{s}}{L_{d}} & n_{p} \omega & 0 \\
-n_{p} \omega & -\frac{R_{s}}{L_{q}} & -\frac{n_{p} \psi_{f}}{L_{q}} \\
0 & \frac{1.5 n_{p} \psi_{f}}{J} & -\frac{B}{J}
\end{array}\right]\left[\begin{array}{c}
i_{d} \\
i_{q} \\
\omega_{r}
\end{array}\right]+\left[\begin{array}{c}
\frac{u_{d}}{L_{d}} \\
u_{q} \\
\frac{L_{q}}{T_{L}} \\
-\frac{T_{L}}{J}
\end{array}\right],
$$

where $R_{s}$ is the stator resistance; $L_{d}$ and $L_{q}$ are stator inductances and $L_{d}=L_{q}=L ; \psi_{f}$ is the rotor flux linkage; $u_{d}$ and $u_{q}$ are stator voltages; $i_{d}$ and $i_{q}$ are stator currents; $\omega_{r}$ is the rotor angular velocity; $J$ is the moment of inertia; $B$ is the viscous friction coefficient; $n_{p}$ is the number of pole pairs, and $T_{L}$ is the load torque.

In the previous vector control design of PMSM, the $q$ axes stator current $i_{q}$ is usually approximately replaced by the $q$-axes reference current $i_{q}^{*}$, which degrades the closed-loop system performance. Motivated by the built second-order model of PMSM in [28], we propose a fractional-order model between the $q$-axes reference current $i_{q}^{*}$ and the position output.

Considering the input and output of the current loop $i_{q}$ in Figure 1, the following Laplace-transform equation can be easily obtained:

$$
\frac{U_{q}(s)}{I_{q}^{*}(s)-I_{q}(s)}=k_{p}+\frac{k_{i}}{s^{\lambda}},
$$

where $U_{q}(s), I_{q}^{*}(s)$, and $I_{q}(s)$ are the Laplace transformations of $u_{q}, i_{q}^{*}$, and $i_{q}$, respectively; $k_{p}$ and $k_{i}$ are the proportional and integral gains of the $\mathrm{PI}^{\lambda}$ controller in the current loop $i_{q}$, respectively; $\lambda$ is the power of $s$ in integral actions.

The fractional-order PMSM dynamic equation is derived as

$$
\ddot{\omega}=-\frac{k_{i}}{k_{p}} \omega^{(2-\lambda)}+d(t)+u,
$$

where $d(t)=-\left(b / k_{p}\right) \dot{u}_{q}+\dot{a}(t)+\left(k_{i} / k_{p}\right) a^{(1-\lambda)}(t) ; b=$ $1.5 n_{p} \psi_{f} / J ; a(t)=-(B / J) \omega-\left(T_{L} / J\right) ; u=b\left(i_{q}^{*}+\left(k_{i} / k_{p}\right) i_{q}^{*}(1-\lambda)\right)$; $d(t)$ represents the lumped disturbances including viscous frictions and external load disturbances.

For the convenience of designing the controller, assume that the speed control loop, the current control loop, and the inverter are ideal [29]. Thus, the fractional-order model of PMSM position regulation system is described by

$$
\begin{gathered}
\dot{\theta}=\omega, \\
\ddot{\omega}=-\frac{k_{i}}{k_{p}} \dot{\omega}^{(1-\lambda)}+d(t)+u .
\end{gathered}
$$

The tracking error can be defined as follows:

$$
e=\theta-\theta_{d} \text {. }
$$

The control objective is to design a F2NTSMC with SMDO to track the reference trajectory $\theta_{d}$ in finite time. In the next section, the design of F2NTSMC and SMDO will be conducted.

\section{Control Design}

In this section, a fractional-order nonsingular fast terminal sliding mode controller is proposed to achieve equivalence between fast convergence and nonsingularity. The first step is to develop the F2NTSMC to achieve chattering-free and robust tracking of the position. And then, a sliding mode disturbance observer is designed to estimate and compensate uncertainties and disturbances, which can increase robustness of the control system and improve control performance. Thus, a control scheme with F2NTSMC and SMDO is presented.

4.1. Fractional Second-Order Nonsingular Terminal Sliding Mode Control. To compare the convergence performance between TSMC and NTSMC, the following sliding mode surfaces are considered:

$$
\begin{gathered}
s_{1}(t)=D^{r_{1}} x+a_{1} x+a_{2} \operatorname{sig}^{\lambda_{1}}(x), \\
s_{2}(t)=D^{r_{2}} x+a_{3} x+a_{4} \operatorname{sig}^{\lambda_{2}}(\dot{x}), \\
s_{3}(t)=D^{r_{3}} x+a_{5} \operatorname{sig}^{\lambda_{3}}(x)+a_{6} \operatorname{sig}^{\lambda_{4}}(x), \\
s_{4}(t)=D^{r_{4}} x+a_{7} \operatorname{sig}^{\lambda_{5}}(x)+a_{8} \operatorname{sig}^{\lambda_{6}}(\dot{x}),
\end{gathered}
$$




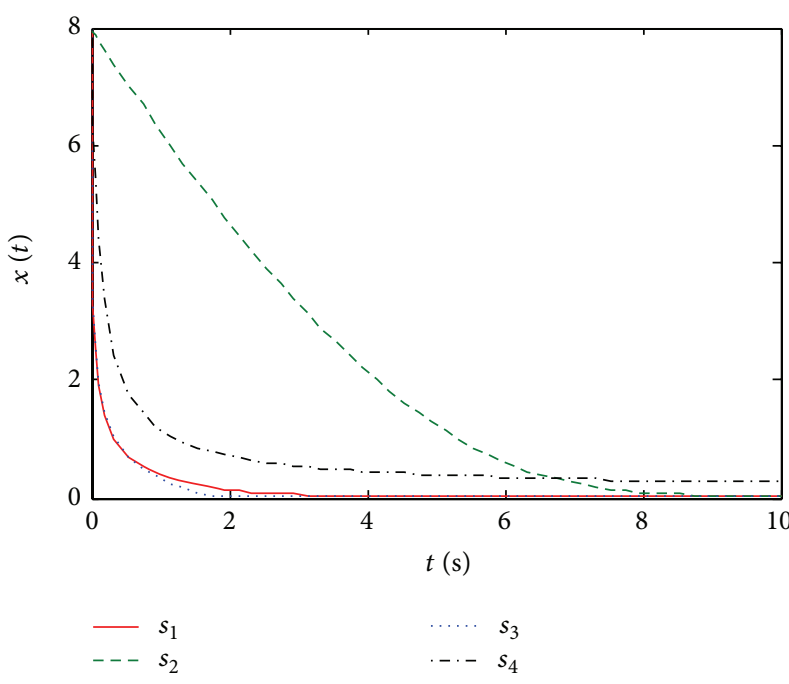

(a)

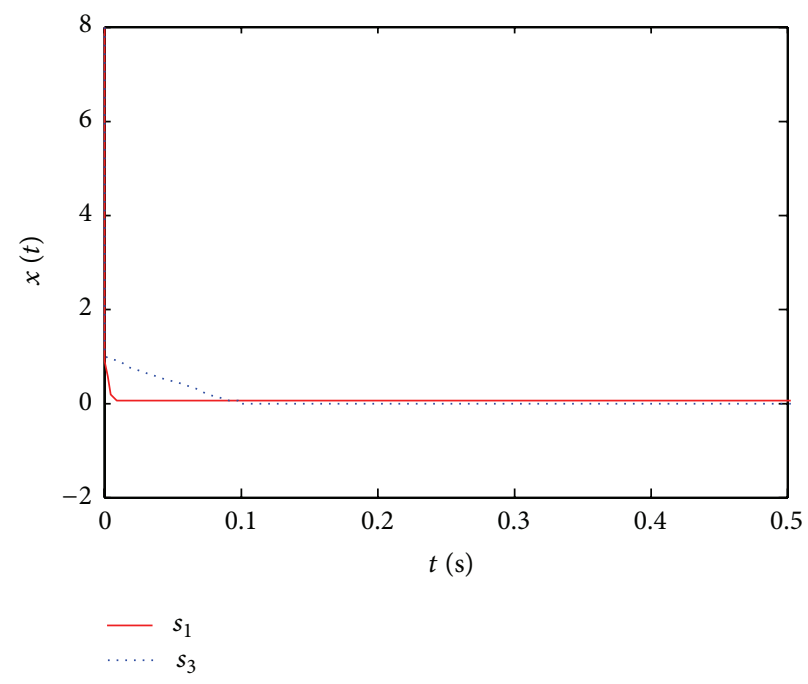

(b)

FIGURE 2: Convergence conditions of $s_{1, \ldots, 4}$.

where $r_{i} \in(0,1], i=1, \ldots, 4 ; a_{j}>0, j=1, \ldots, 8$; $\lambda_{1}>0, \lambda_{2}>1, \lambda_{3}>1,0<\lambda_{4}<1, \lambda_{5}>\lambda_{6}$, and $1<\lambda_{6}<2 ; \operatorname{sig}^{\lambda}(e)=|e|^{\lambda} \operatorname{sign}(e)$ and $s_{1}(t), s_{3}(t)$ are FTSM surfaces, and $s_{2}(t), s_{4}(t)$ are NFTSM surfaces. All their initial states are set as $x(0)=8$. The parameters are given by $a_{i}=1, \quad i=1, \ldots, 8, \lambda_{1,2,3,5}=3, \lambda_{4}=0.5$, and $\lambda_{6}=1.5$. It can be seen from Figure 2 that $s_{1}(t)$ and $s_{3}(t)$ have faster convergence rate than $s_{2}(t)$ and $s_{4}(t)$. For the more detailed comparison between $s_{1}(t)$ and $s_{3}(t)$, we consider parameters $a_{1,2,5,6} \neq 1$. The parameters of $s_{1}(t)$ and $s_{3}(t)$ are tuned with the optimal integrated time absolute error (ITAE) criterion by minimizing the following formula:

$$
J_{\mathrm{ITAE}}=\int_{t_{0}}^{\infty} t|x(t)| d t .
$$

The optimal parameters of $s_{1}(t)$ and $s_{3}(t)$ are $\left[\lambda_{1}, a_{1}, a_{2}\right]^{\prime}=[47.65,304.19,80.83]^{\prime}$ and $\left[\lambda_{3}, \lambda_{4}, a_{5}, a_{6}\right]^{\prime}$ $=[242.69,31.25,218.75,1]^{\prime}$, respectively. The convergence rate of $s_{1}(t)$ is higher than its counterpart of $s_{3}(t)$.

Therefore, the fractional-order sliding mode surface $s_{1}(t)$ is chosen to be researched in this paper and rewritten as

$$
s(t)=D^{r} e+b_{1} e+b_{2} \operatorname{sig}^{z}(e),
$$

where $0<r<1, b_{1}, b_{2}>0$, and $z \geq 2$. Note that

$$
\begin{gathered}
\frac{d}{d t} \operatorname{sig}^{z}(e)=z|e|^{z-1} \dot{e} \\
\frac{d^{2}}{d t^{2}} \operatorname{sig}^{z}(e)=z(z-1) \operatorname{sig}^{z-2}(e) \dot{e}^{2}+z|e|^{z-1} \ddot{e} .
\end{gathered}
$$

Once the system operates in the sliding manifold, equation $s(t)=0$ is satisfied. Since $s(t)=0$ holds in (13), it follows that

$$
D^{r} e=-b_{1} e-b_{2} \operatorname{sig}^{z}(e) .
$$

Then, a nonsingular fast terminal sliding surface [29] is selected to ensure $s(t)$ reach zero in finite time and realize second-order sliding mode control:

$$
\sigma=s+\alpha s^{g / h}+\beta \dot{s}^{p / q},
$$

where $\alpha, \beta>0, g, h, p, q$ are positive odd integers, $g / h>1$, and $1<p / q<2$.

Furthermore, the following continuous terminal sliding mode reaching law [29] is introduced to guarantee system states converge to sliding surfaces in finite time and increase system robustness:

$$
\dot{\sigma}=\left(-\phi \sigma-\gamma \sigma^{m / n}\right) \dot{s}^{p / q-1}
$$

where $\phi, \gamma>0, m, n$ are positive odd integers and $0<m / n<$ 1.

Suppose $t_{r_{1}}$ is the time when $\sigma$ reaches zero from $\sigma(0) \neq 0$, that is $\sigma(t)=0$ when $t \geq t_{r_{1}}$. Solving (17) as [29], the time from $\sigma(0) \neq 0$ to $s\left(t_{s_{1}}\right)=0$ can be obtained as follows:

$$
\begin{aligned}
t_{s_{1}}= & t_{r_{1}}+2 \tau_{1}^{-q / p} \frac{p}{p-q} V(0)^{(p-q) / 2 p} \\
& \times F\left(A, B, C,-\frac{\tau_{2}}{\tau_{1}} V(0)^{(g-h) / 2 h}\right),
\end{aligned}
$$

where $\tau_{1}=2^{(p+q) / 2 q}(1 / \beta), \tau_{2}=2^{p / 2 q+g / 2 h}(\alpha / \beta), A=q / p$, $B=h(p-q) / p(g-h), C=(p g-q h) / p(g-h), F(\cdot)$ is Gauss hypergeometric function, and the Lyapunov function is $V(t)=(1 / 2) s^{2}$.

Thus, the second-order sliding mode control is achieved. $s$ and $\dot{s}$ are driven to reach $\sigma=0$ in finite time and then remain on $\sigma=0$ to realize the sliding mode motion. Both $s$ and $\dot{s}$ reach zero in finite time $t_{s_{1}}$. After $s$ reaches zero, the system will stay on the sliding mode motion (13) and the tracking error $e$ will converge to zero in finite time $t_{s_{0}}$ which 
is calculated in Theorem 5. Next, the second-order sliding mode controller will be derived.

Take the second time derivative of both sides of (13), one obtains

$$
\ddot{s}(t)=D^{r-1} \ddot{e}+b_{1} \ddot{e}+b_{2} E,
$$

where $E=z(z-1) \operatorname{sig}^{z-2}(e) \dot{e}^{2}+z|e|^{z-1} \ddot{e}$.

Differentiating the sliding variable $\sigma,(16)$ gets

$$
\dot{\sigma}=\dot{s}+\frac{\alpha g}{h} s^{g / h-1} \dot{s}+\frac{\beta p}{q} \dot{s}^{p / q-1} \ddot{s} .
$$

Substituting (19) into (20), it follows that

$$
\dot{\sigma}=\varphi+\Delta+\eta D^{r-1} u,
$$

where $\eta=(\beta p / q) \dot{s}^{p / q-1}, \Lambda=b_{1} \ddot{e}+b_{2} E-D^{r-1}\left(\ddot{\theta}_{d}+\right.$ $\left.\left(k_{i} / k_{p}\right) \ddot{\theta}^{(1-\lambda)}\right), \varphi=\dot{s}+(\alpha g / h) s^{g / h-1} \dot{s}+\eta \Lambda$, and $\Delta=\eta D^{r-1} d(t)$.

From (17) and (21), the fractional-order terminal sliding mode controller is designed as

$$
\begin{gathered}
u=-\eta^{-1} D^{1-r}\left[\left(\phi \sigma+\gamma \sigma^{m / n}\right) \dot{s}^{p / q-1}+\varphi+\Delta\right] \\
=-\frac{q}{\beta p} D^{1-r}\left[\phi \sigma+\gamma \sigma^{m / n}+\left(1+\frac{\alpha g}{h} s^{g / h-1}\right)\right. \\
\left.\times \dot{s}^{2-p / q}+\frac{\beta p}{q} \Lambda\right]-d(t),
\end{gathered}
$$

where $d(t)$ denotes unknown uncertainties and disturbances.

Considering (22), all fractional powers of $s$ and $\dot{s}$ are positive; that is, the control method is nonsingular. After the system state enters the sliding mode $\sigma=0$, there is also no singularity. Therefore, the system is globally nonsingular during both the reaching phase and the sliding phase.

4.2. Sliding Mode Disturbance Observer. In order to increase robustness of the controller and improve control performance, a SMDO is proposed to estimate uncertainties and disturbances.

Equation (21) is rewritten as

$$
\dot{\bar{\sigma}}=\bar{\varphi}+d+u
$$

where $\dot{\bar{\sigma}}=\eta^{-1} D^{1-r} \dot{\sigma}$ and $\bar{\varphi}=\eta^{-1} D^{1-r} \varphi$.

Select the auxiliary sliding variable as

$$
\rho=\bar{\sigma}-\lambda \text {. }
$$

Construct a disturbance observer as follows:

$$
\dot{\lambda}=\bar{\varphi}+u+v .
$$

By differentiating $\rho$ with respect to time, one obtains

$$
\dot{\rho}=d-v \text {. }
$$

Then, the auxiliary super-twisting sliding mode control is designed to drive the sliding variable $\rho$ to zero:

$$
\begin{gathered}
v=k_{o_{1}}|\rho|^{3 / 2} \operatorname{sign}(\rho)-z, \\
\dot{z}=-k_{o_{2}} \rho^{2} \operatorname{sign}(\rho) .
\end{gathered}
$$

Equation (26) closed by control (27) results in

$$
\begin{gathered}
\dot{\rho}=-k_{o_{1}}|\rho|^{3 / 2} \operatorname{sign}(\rho)+z+d, \\
\dot{z}=-k_{o_{2}} \rho^{2} \operatorname{sign}(\rho) .
\end{gathered}
$$

Theorem 4. Suppose that the uncertainties of the system (28) are globally bounded by

$$
|d| \leq \delta|\rho|^{3 / 2}
$$

Then, sliding variable $\rho$ of (28) converges in finite time to the origin $\rho=0$ if the gains satisfy the following relations:

$$
k_{o_{1}}>2 \delta, \quad k_{o_{2}}>\frac{k_{o_{1}} \delta^{2}}{8\left(k_{o_{1}}-2 \delta\right)} .
$$

The convergence time $t\left(\rho_{0}\right)$ is upperbounded by $\bar{T}=2 V^{1 / 2}\left(\rho_{0}\right) / \bar{\gamma}$, where $\rho_{0}$ is the initial state and $\bar{\gamma}=\lambda_{\min }^{1 / 2}(\chi) \lambda_{\min }(\bar{\Theta}) / \lambda_{\max }(\chi)[30]$.

Proof. We propose the following Lyapunov function and its quadratic form:

$$
\begin{aligned}
V= & k_{o_{2}}|\rho|^{3}+\left(D^{-1} z\right)^{2} \\
& +\frac{1}{2}\left(k_{o_{1}}|\rho|^{3 / 2} \operatorname{sign}(\rho)-D^{-1} z\right)^{2} \\
= & \zeta^{T} \chi \zeta,
\end{aligned}
$$

where

$$
\begin{gathered}
\zeta^{T}=\left[|\rho|^{3 / 2} \operatorname{sign}(\rho), D^{-1} z\right], \\
\chi=\frac{1}{2}\left[\begin{array}{cc}
k_{o_{1}}^{2}+2 k_{o_{2}} & -k_{o_{1}} \\
-k_{o_{1}} & 3
\end{array}\right] .
\end{gathered}
$$

Its time derivative along the solution of (31) is

$$
\dot{V}=-|\rho|^{1 / 2} \zeta^{T} \Theta \zeta+|\rho|^{1 / 2} d q_{1}^{T} \zeta,
$$

where

$$
\begin{gathered}
\Theta=\frac{3 k_{o_{1}}}{2}\left[\begin{array}{cc}
k_{o_{1}}^{2}+\frac{4}{3} k_{o_{2}} & -k_{o_{1}} \\
-k_{o_{1}} & 1
\end{array}\right], \\
q_{1}^{T}=3\left[\frac{k_{o_{1}}^{2}}{2}+k_{o_{2}},-\frac{k_{o_{1}}}{2}\right] .
\end{gathered}
$$

Applying (29), it yields

$$
\dot{V} \leq-|\rho|^{1 / 2} \zeta^{T} \bar{\Theta} \zeta
$$

where

$$
\bar{\Theta}=\frac{3 k_{o_{1}}}{2}\left[\begin{array}{cc}
k_{o_{1}}^{2}+\frac{4}{3} k_{o_{2}}-\left(\frac{2 k_{o_{2}}}{k_{o_{1}}}+k_{o_{1}}\right) \delta & -k_{o_{1}}+\frac{\delta}{2} \\
-k_{o_{1}}+\frac{\delta}{2} & 1
\end{array}\right] .
$$


$\dot{V}$ is negative definite if $\bar{\Theta}>0$. It is easy to see that this is the case if the gains are as in (30). The sliding variable $\rho$ is driven to zero in finite time $\bar{T}=2 V^{1 / 2}\left(\rho_{0}\right) / \bar{\gamma}$. Namely, the lumped disturbances $d(t)$ are exactly estimated by $v=\widehat{d}$ in finite time $\bar{T}$. This completes the proof.

Eventually, the fractional-order terminal sliding mode controller with SMDO is designed as

$$
\begin{gathered}
u=-\eta^{-1} D^{1-r}\left[\left(\phi \sigma+\gamma \sigma^{m / n}\right) \dot{s}^{p / q-1}+\varphi+\Delta\right] \\
=-\frac{q}{\beta p} D^{1-r}\left[\phi \sigma+\gamma \sigma^{m / n}+\left(1+\frac{\alpha g}{h} s^{g / h-1}\right)\right. \\
\left.\times \dot{s}^{2-p / q}+\frac{\beta p}{q} \Lambda\right]-\widehat{d}(t) .
\end{gathered}
$$

In the next section, the stability of the proposed controller will be proved.

4.3. Stability Analysis. The stability analysis consists of two parts. The first part is to prove that position tracking error (10) of the system (9) converges to $e=0$ in finite time. The second part is to verify whether or not the proposed approach (37) can ensure the system trajectories (21) converge to the sliding surface $s(t)=0$ in finite time and have no singularity.

Theorem 5. Consider the sliding mode surface (13). The tracking errors converge to the origin $e=0$ in finite time $t_{s_{0}} \leq\left(V^{r-2}\left(t_{r_{0}}\right) /\left(b_{2}-\ell\right) L\right)^{1 /(2-r)}$, where $t_{r_{0}}$ is the reaching time from $s \neq 0$ to $s=0, b_{2}$, and $\ell$ and $L$ are positive constants.

Proof. Motivated by [25, 31], assume that the following inequality is valid:

$$
\left|\sum_{j=1}^{\infty} \frac{\Gamma(r+1)}{\Gamma(j+1) \Gamma(r-j+1)} D^{j} e D^{r-j} e\right| \leq \ell|e|^{\zeta},
$$

where $\ell$ and $\zeta$ are optional positive constants.

The Lyapunov function is defined as

$$
V(t)=e^{2}
$$

Taking fractional-order derivative of both sides of (39) with respect to time, it yields [31]

$$
D^{r} V(t)=e D^{r} e+\sum_{j=1}^{\infty} \frac{\Gamma(r+1)}{\Gamma(j+1) \Gamma(r-j+1)} D^{j} e D^{r-j} e .
$$

The value of $\zeta$ is set as $(z+1)$. Substituting (15) and inequality (38) into (40), one has

$$
\begin{aligned}
D^{r} V(t) & \leq e D^{r} e+\left|\sum_{j=1}^{\infty} \frac{\Gamma(r+1)}{\Gamma(j+1) \Gamma(r-j+1)} D^{j} e D^{r-j} e\right| \\
& \leq-e\left(b_{1} e+b_{2} \operatorname{sig}^{z}(e)\right)+\ell|e|^{z+1} \leq-\left(b_{2}-\ell\right)|e|^{z+1},
\end{aligned}
$$

where $b_{2}$ is chosen appropriately such that $b_{2}>\ell$ is satisfied. Consequently, by Theorem 5 , the system error $e(t)$ will converge to zero asymptotically. Next, the convergence of $e(t)$ to zero in finite time will be proved.

Taking fractional-order integral of (38) from reaching time $t_{r_{0}}$ to stopping time $t_{s_{0}}$, one obtains

$$
V\left(t_{s_{0}}\right)-V^{r-2}\left(t_{r_{0}}\right) \frac{t_{s_{0}}^{r-2}}{\Gamma(r-1)} \leq-\left(b_{2}-\ell\right) D^{1-r}|e|^{z+1} .
$$

According to [25], there exists a positive constant $L$ such that $D^{-r}|e|^{z+1} \geq L$. Noting that $e\left(t_{s_{0}}\right)=0$, it follows that

$$
-V^{r-2}\left(t_{r_{0}}\right) \frac{t_{s_{0}}^{r-2}}{\Gamma(r-1)} \leq-\left(b_{2}-\ell\right) L .
$$

Solving the inequality (43), it yields

$$
t_{s_{0}} \leq\left(\frac{V^{r-2}\left(t_{r_{0}}\right)}{\left(b_{2}-\ell\right) L}\right)^{1 /(2-r)} .
$$

Therefore, the tracking error $e$ (10) will converge to zero in finite time. This completes the proof.

Theorem 6. Consider the fractional-order PMSM system (9). If the system is controlled by the control input (37), then system states will converge to the sliding surface $s(t)=0$ in finite time $t_{s_{1}}(18)$.

Proof. Consider the following Lyapunov function

$$
V=\frac{1}{2} \sigma^{2}
$$

Differentiating $V$ with respect to time, we have

$$
\begin{aligned}
& \dot{V}=\sigma \dot{\sigma}=\sigma\left(\varphi+\Delta+\eta D^{r-1} u\right) \\
&=\sigma\{\varphi+\Delta+\eta \\
& \times\left[\eta ^ { - 1 } \left(-\varphi-\left(\phi \sigma+\gamma \sigma^{m / n}\right)\right.\right. \\
&\left.\left.\left.\times \dot{s}^{p / q-1}-D^{r-1} \widehat{d}(t)\right)\right]\right\} \\
&=\sigma\left(-\left(\phi \sigma+\gamma \sigma^{m / n}\right) \dot{s}^{p / q-1}+\Delta-\widehat{\Delta}\right) \\
&=-\sigma\left(\phi \sigma+\gamma \sigma^{m / n}\right) \dot{s}^{p / q-1}+\sigma \widetilde{\Delta},
\end{aligned}
$$

where $\widetilde{\Delta}=\Delta-\widehat{\Delta}$. Since the sliding variable $\sigma$ converges to zero only after the lumped disturbances $\Delta$ is estimated in finite time $\bar{T}$, that is, $\widetilde{\Delta}=\Delta-\widehat{\Delta} \rightarrow 0$ when $t>\bar{T}$, therefore, for $t>\bar{T}$, we have

$$
\begin{aligned}
\dot{V} & =-\sigma^{T}\left(\phi \sigma+\gamma \sigma^{m / n}\right) \dot{s}^{p / q-1} \\
& \leq-\kappa \dot{s}^{p / q-1}\left(\sigma^{2}+\sigma^{1+m / n}\right) \mid \kappa=\min (\phi, \gamma) \\
& \leq-\kappa \dot{s}^{p / q-1} \sigma^{1+m / n}=-\kappa \dot{s}^{p / q-1} V^{(1+m / n) / 2} .
\end{aligned}
$$

Owing to $0<m / n<1$, it gets that $0<(1+m / n) / 2<1$. 

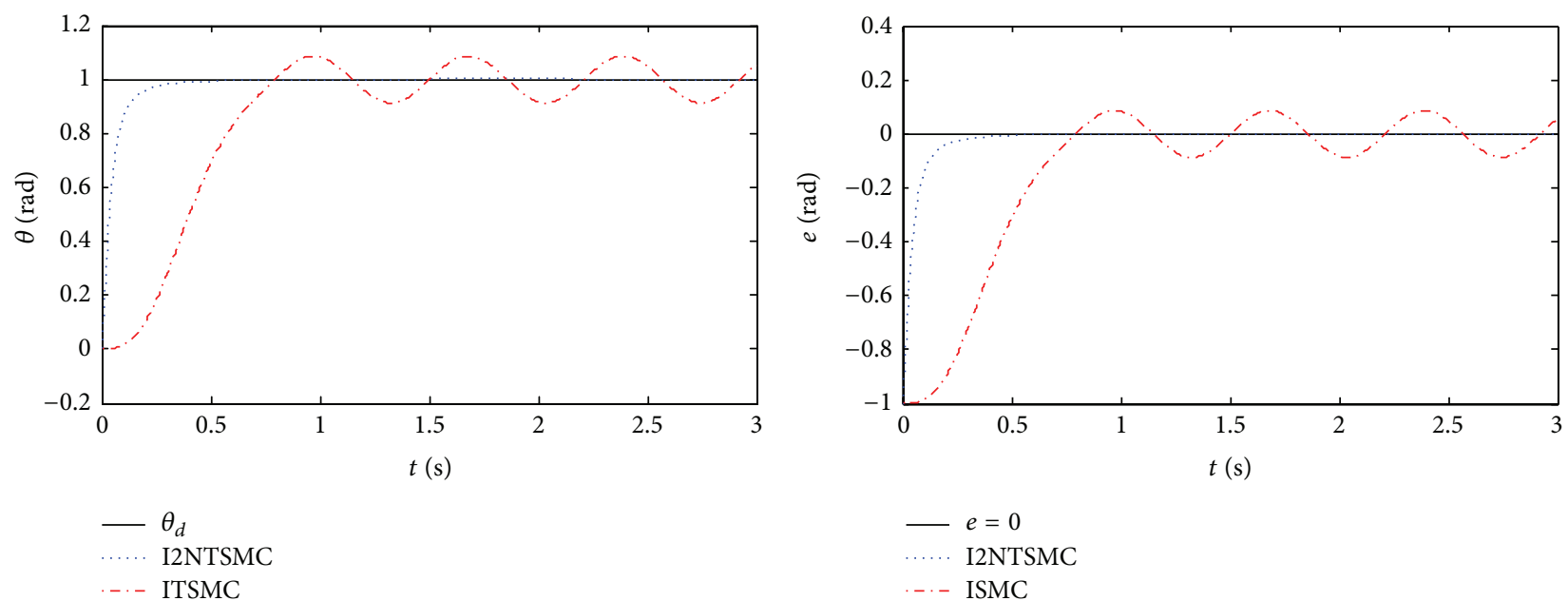

(a)

(b)
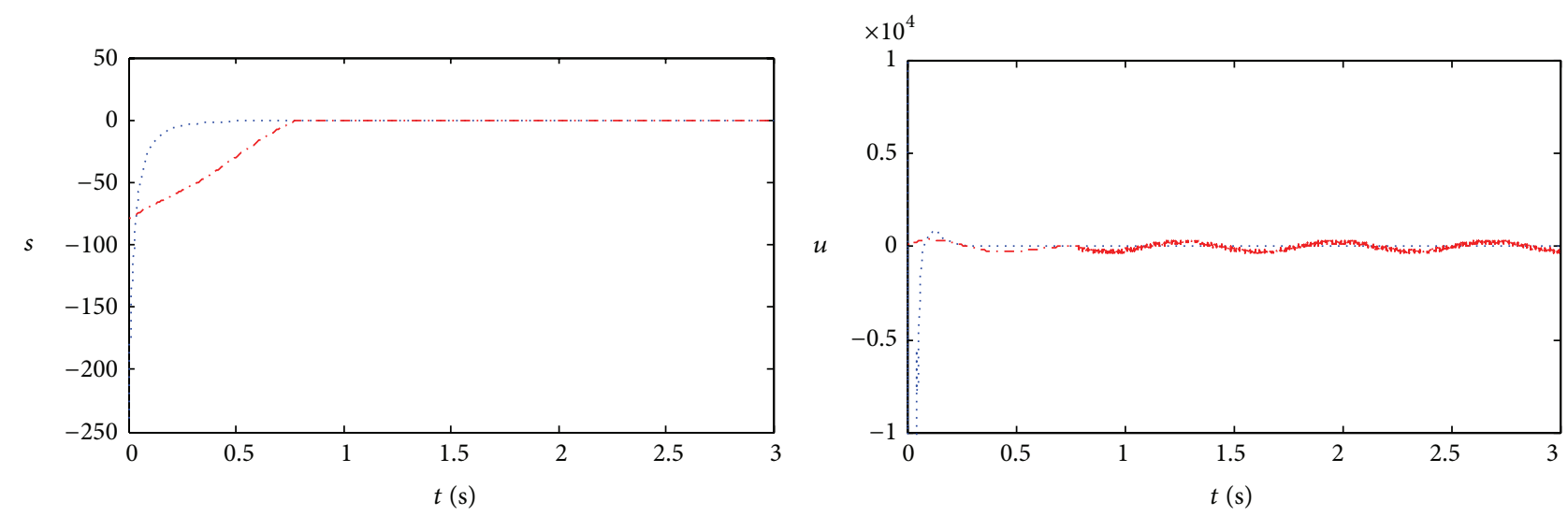

. I2NTSMC

I2NTSMC

-..- ITSMC

... ITSMC

(c)

(d)

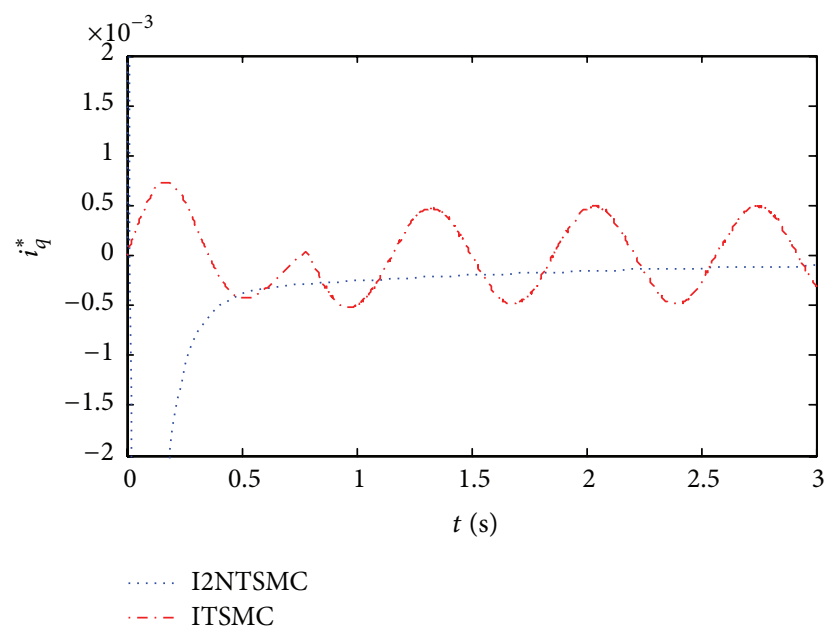

(e)

FIGURE 3: Control performance of ITSMC and I2NTSMC for the nominal system. (a) Position tracking performance, (b) tracking errors, (c) sliding surfaces, (d) control inputs, and (e) current $i_{q}^{*}$. 

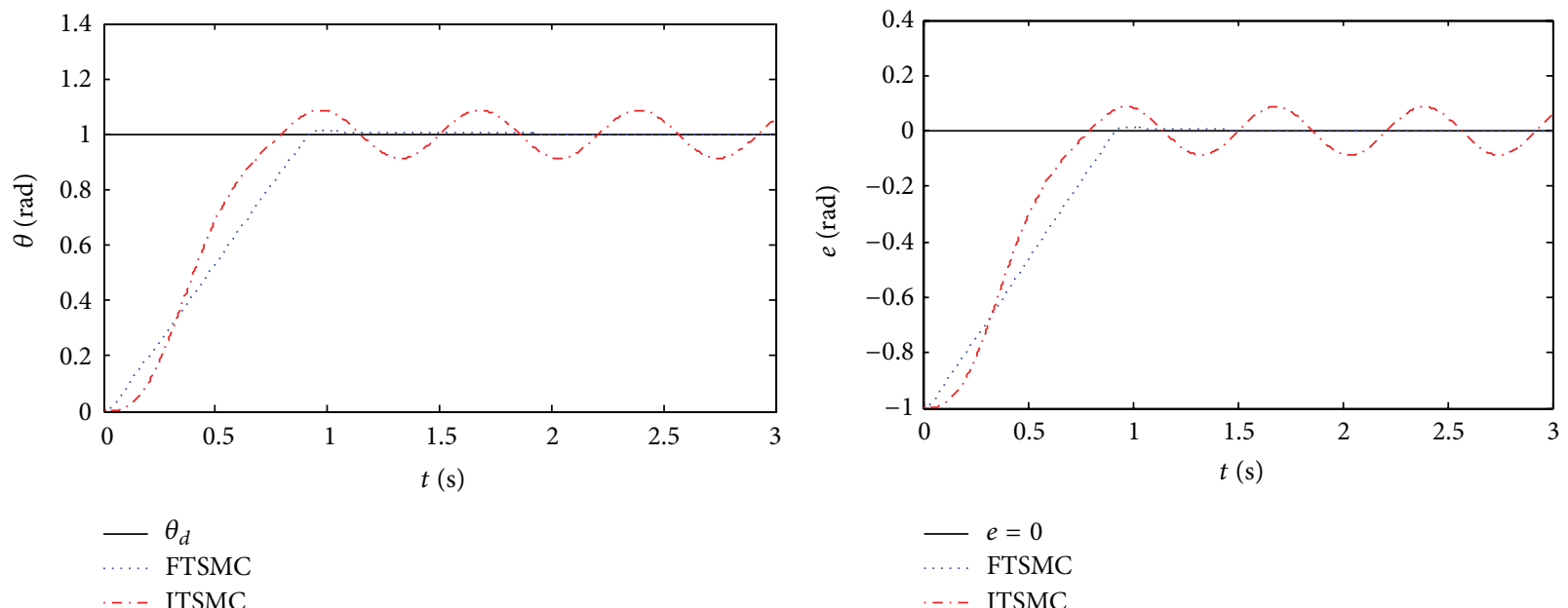

(a)

(b)
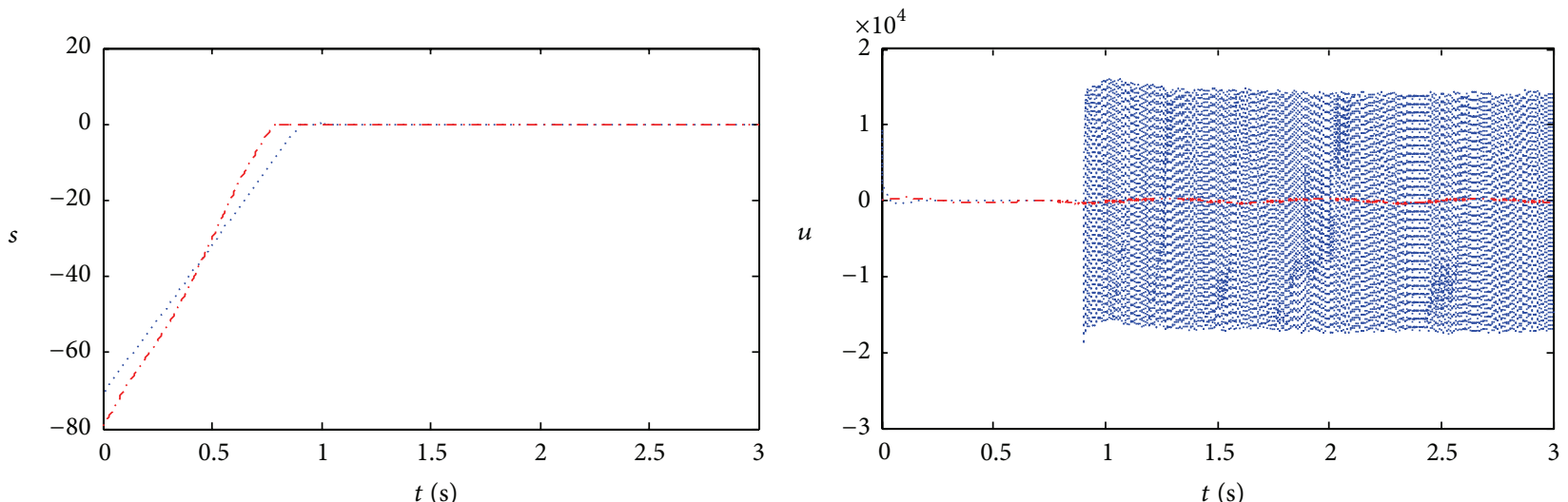

FTSMC

ITSMC

FTSMC

ITSMC

(c)

(d)

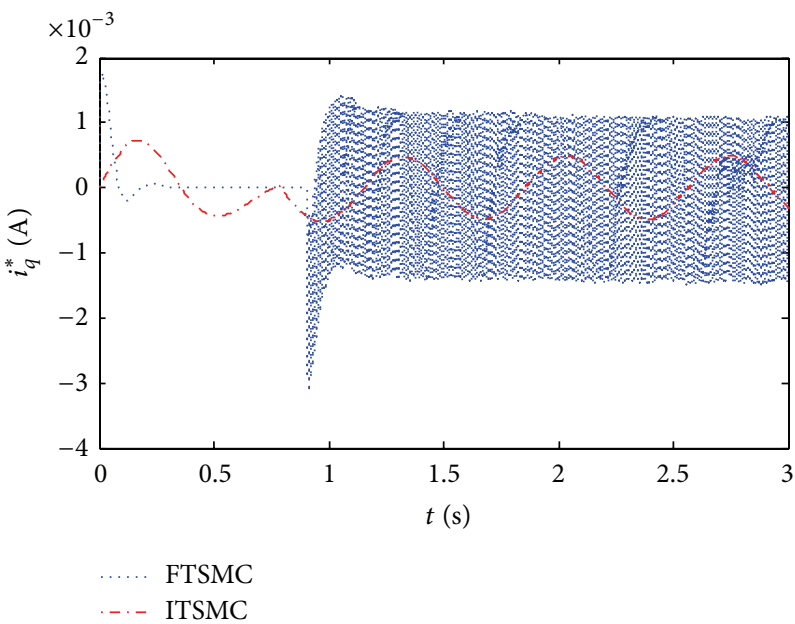

(e)

FIgURE 4: Control performance of ITSMC and FTSMC for the nominal system. (a) Position tracking performance, (b) tracking errors, (c) sliding surfaces, (d) control inputs, and (e) current $i_{q}^{*}$. 

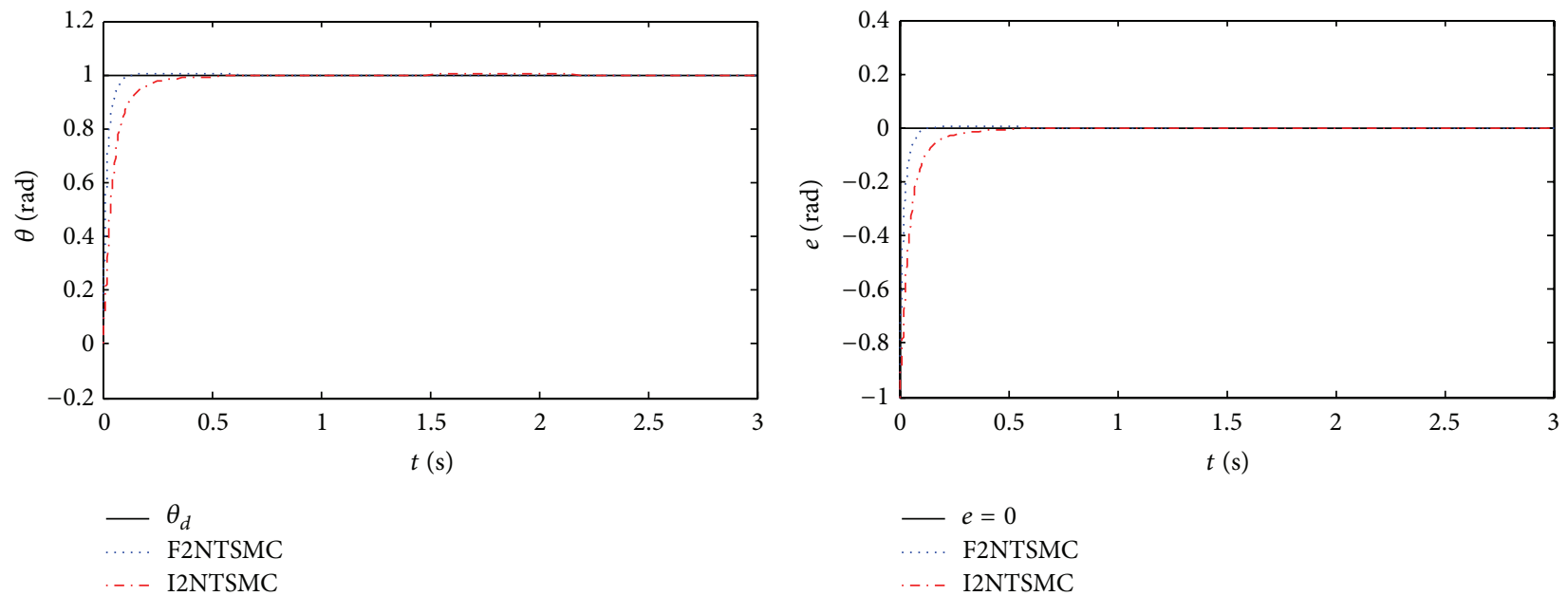

(a)

(b)
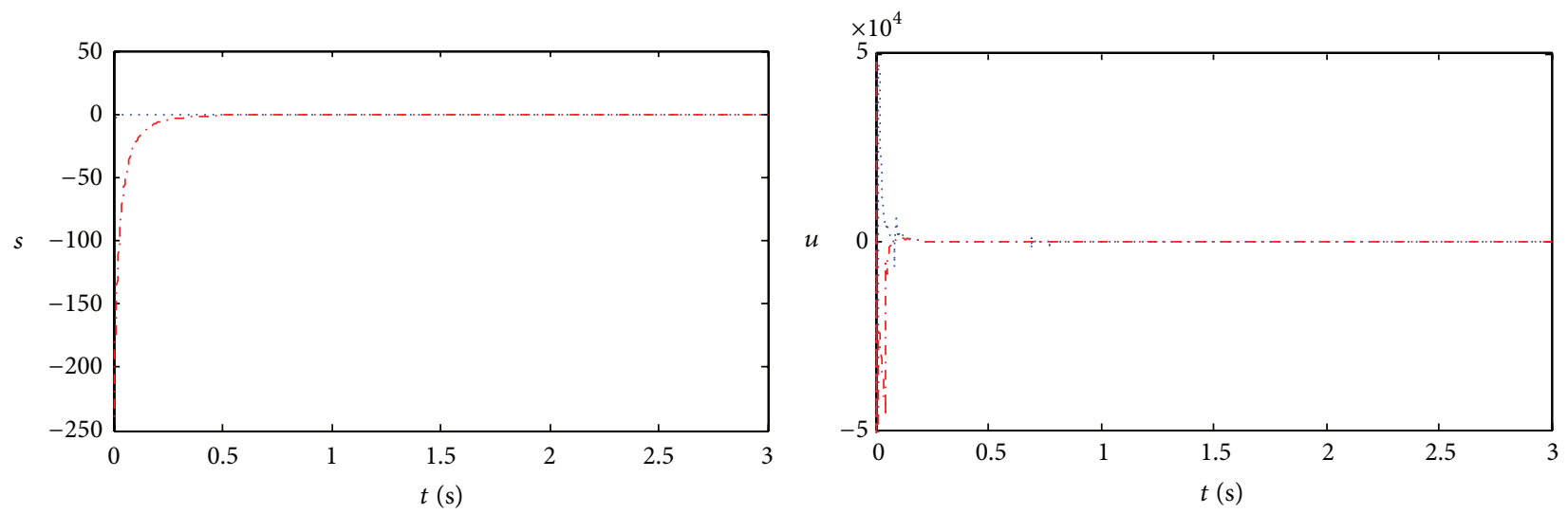

F2NTSMC

F2NTSMC

... I2NTSMC

...- I2NTSMC

(c)

(d)

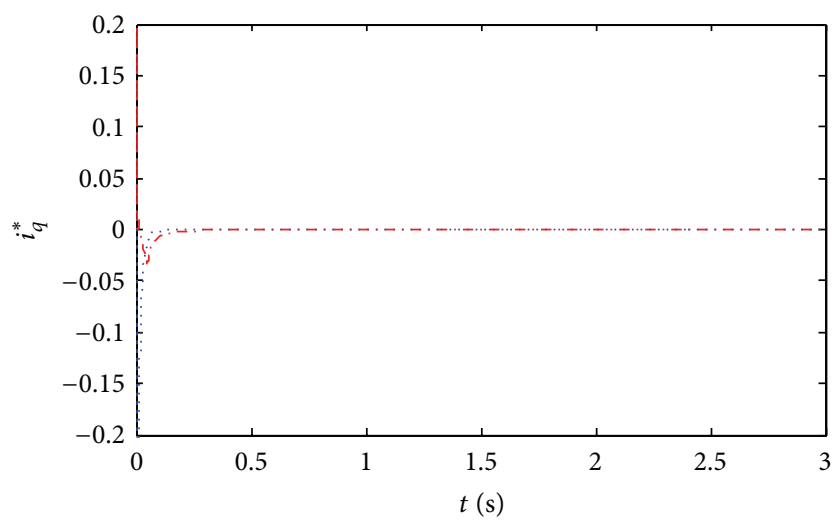

F2NTSMC

-.. I2NTSMC

(e)

FIgURE 5: Control performance of I2NTSMC and F2NTSMC for the nominal system. (a) Position tracking performance, (b) tracking errors, (c) sliding surfaces, (d) control inputs, and (e) current $i_{q}^{*}$. 


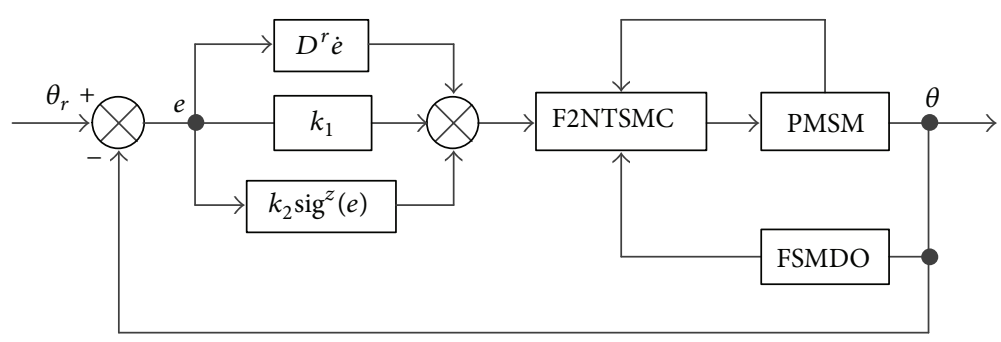

FIGURE 6: Block diagram of the SMDO-based F2NTSMC system.

Equation (47) belongs to the following type of inequality:

$$
\dot{V}(t) \leq-a V^{\eta}(t), \quad \forall t \geq t_{0}, V\left(t_{0}\right) \geq 0 .
$$

Based on [32, Lemma 2], the sliding variables $\sigma$ will converge to zero in finite time driven by the control input (37). Then, $s$ reaches zero in finite time $t_{s_{1}}$. Hence, the proof is achieved.

\section{Simulation Results}

This section presents simulation results to investigate control performance of the proposed method. The parameters of both current loops are set as $k_{p}=200, k_{i}=5000$, and $\lambda=0.2$. The parameter values of the PMSM system are as follows: rated power is $P=7.5 \mathrm{~kW}$; rated voltage is $U=200 \mathrm{~V}$; rated torque is $T_{N}=2.5 \mathrm{~N} \cdot \mathrm{m}$; number of poles is $n_{p}=4$; moment of inertia is $J=1.78 \times 10^{-4} \mathrm{~kg} \cdot \mathrm{m}^{2}$; stator resistance is $R_{s}=1.74 \Omega$; rotor flux linkage is $\psi_{f}=0.402 \mathrm{wb}$; viscous damping coefficient is $B=7.403 \times 10^{-5} \mathrm{~N} \cdot \mathrm{m} \cdot \mathrm{s} / \mathrm{rad}$; rated speed is $n_{N}=3000 \mathrm{rpm}$, and both stator inductances are $L_{q}=L_{d}=4 \mathrm{mh}$. The saturation limit of $i_{q}^{*}$ is $\pm 10 \mathrm{~A}$.

5.1. Simulation of TSMC and 2NTSMC. In this simulation, the performance of the conventional TSMC method and the 2NTSMC method is simulated for comparison. We only consider the tracking control problem for the nominal model, that is, no parameter uncertainties and external disturbances. The integral-order sliding surface of conventional TSMC is designed as follows:

$$
s(t)=\ddot{e}+c_{1} e+c_{2} \operatorname{sig}^{y_{1}}(e),
$$

where $c_{1}$ and $c_{2}$ are set as positive constants and $0<y_{1}<1$.

The constant rate reaching law is chosen as

$$
\dot{s}=-\varepsilon \operatorname{sgn}(s), \quad \varepsilon>0 .
$$

Taking the time derivative on both sides of (49) and substituting (9), (10), and (49) into (50), the conventional sliding mode control input can be obtained as

$$
u_{\mathrm{SMC}}=-c_{1} \dot{e}-c_{2} y_{1}|e|^{y_{1}-1} \dot{e}-\varepsilon \operatorname{sgn}(s)+\dddot{\theta}_{d}+\frac{k_{i}}{k_{p}} \ddot{\theta}^{(1-\lambda)} \text {. }
$$

The integral-order sliding surface of 2NTSMC is designed as follows:

$$
s(t)=\dot{e}+c_{3} e+c_{4} \operatorname{sig}^{y_{2}}(e) .
$$

Taking the second derivatives with respect to time on both sides of (52) and substituting (9), (10), and (52) into (17) and (20), the control input of 2NTSMC can be gotten as

$$
\begin{aligned}
u_{2 \mathrm{NTSMC}}= & -\frac{q}{\beta p}\left[\phi \sigma+\gamma \sigma^{m / n}+\left(1+\frac{\alpha g}{h} s^{g / h-1}\right) \dot{s}^{2-p / q}\right] \\
& -\Lambda^{\prime}-d(t),
\end{aligned}
$$

where

$$
\begin{gathered}
\Lambda^{\prime}=c_{3} \ddot{e}+c_{4} E-\left(\dddot{\theta}_{d}+\frac{k_{i}}{k_{p}} \ddot{\theta}^{(1-\lambda)}\right), \\
E=y_{2}\left(y_{2}-1\right) \operatorname{sig}^{y_{2}-2}(e) \dot{e}^{2}+y_{2}|e|^{y_{2}-1} \ddot{e} .
\end{gathered}
$$

The following control parameters are all tuned with the optimal integrated time absolute error (ITAE) criterion.

For the nominal system, the optimal control parameters in (51) are selected as $c_{1}=78.48, c_{2}=0.84, y_{1}=190.71$, and $\varepsilon=95.81$. The optimal integral-order 2NTSMC (I2NTSMC) parameters in (53) are selected as $p=5, q=3, g=7, h=3$, $m=1, n=3, c_{3}=179.77, c_{4}=59.78, y_{2}=6.53, \alpha=80.32$, $\beta=6.95, \phi=73.99$, and $\gamma=59.62$. Simulation results are shown in Figure 3, respectively.

Figure 3 displays time diagrams of position tracking performance, tracking errors, sliding surfaces, control inputs, and current $i_{q}^{*}$, respectively. The PMSM system controlled by both I2NTSM and ITSM controllers is stable. The control input $u_{2 \mathrm{NTSMC}}$ is continuous and chattering-free from Figure 3(d). In comparison with the ITSMC, the I2NTSMC can improve the transient performance substantially and offer higher tracking precision as seen in Figures 3(a), 3(b) and 3(c). It is because that I2NTSMC combines advantages of ITSMC and those of second-order SMC, which increases the convergence rate of the PMSM system and tracking precision.

5.2. Simulation of ITSMC and FTSMC. For comparison between ITSMC and FTSMC, we design the following fractional order sliding surface as

$$
s=D^{r} \dot{e}+c_{3} e+c_{4} \operatorname{sig}^{y_{2}}(e) .
$$



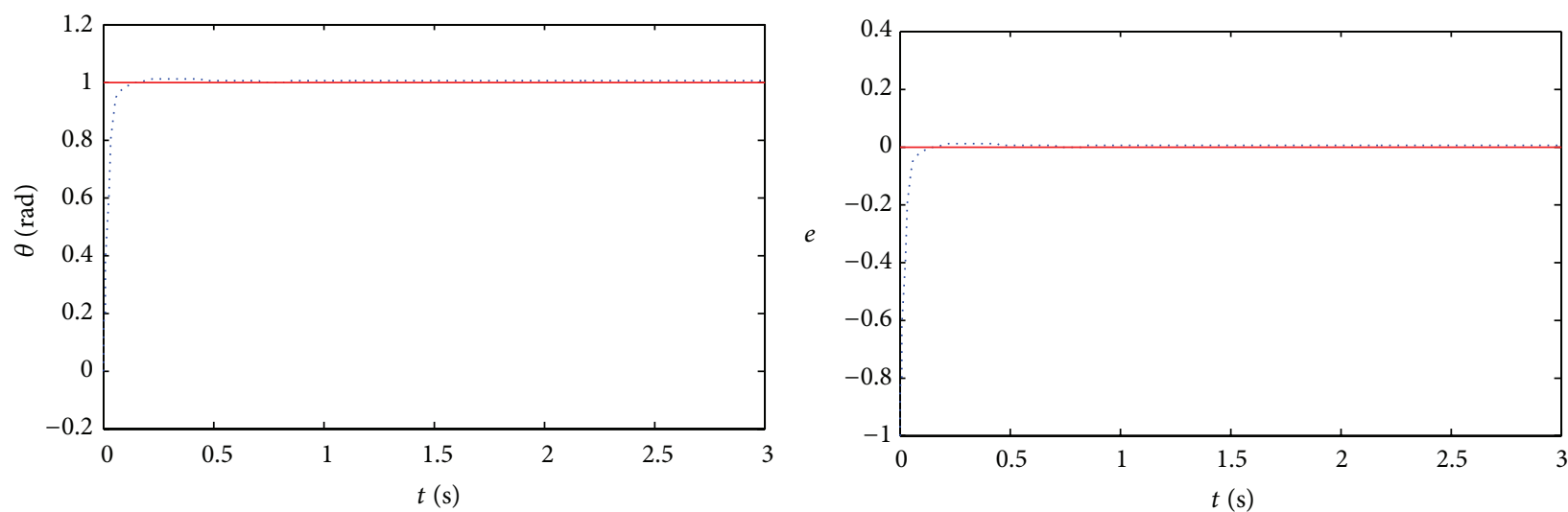

F2NTSMC with $d(t)$

$$
\theta_{d}
$$

(a)

F2NTSMC with $d(\mathrm{t})$

$e=0$

(b)

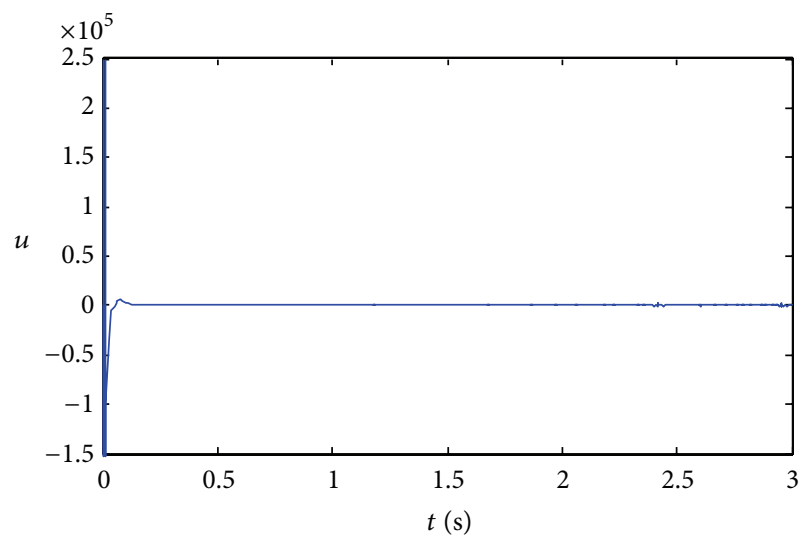

— F2NTSMC with $d(t)$

(d)

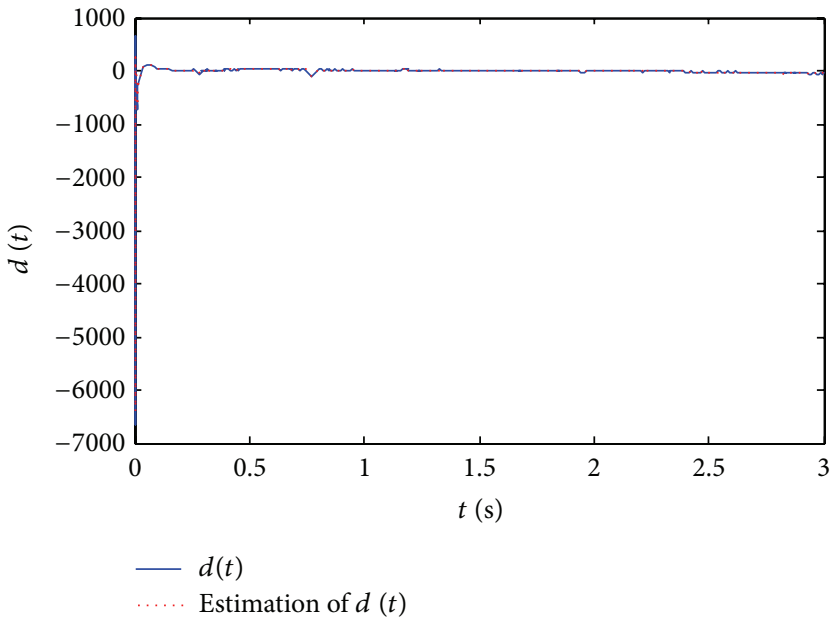

(f)

Figure 7: SMDO-based F2NTSMC for the system with $d(t)$. (a) Position tracking performance, (b) tracking errors, (c) sliding surfaces, (d) control inputs, (e) current $i_{q}^{*}$, and (f) lumped disturbances and their estimation values. 


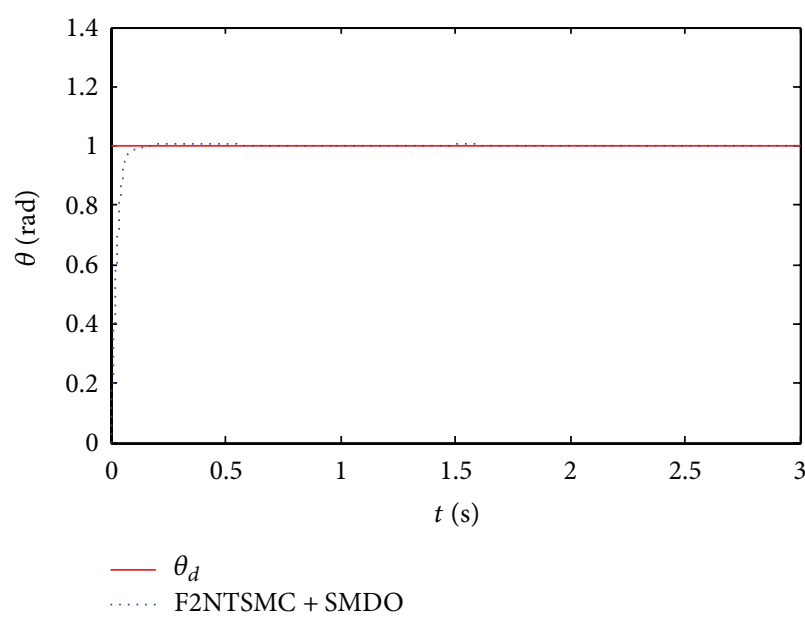

(a)

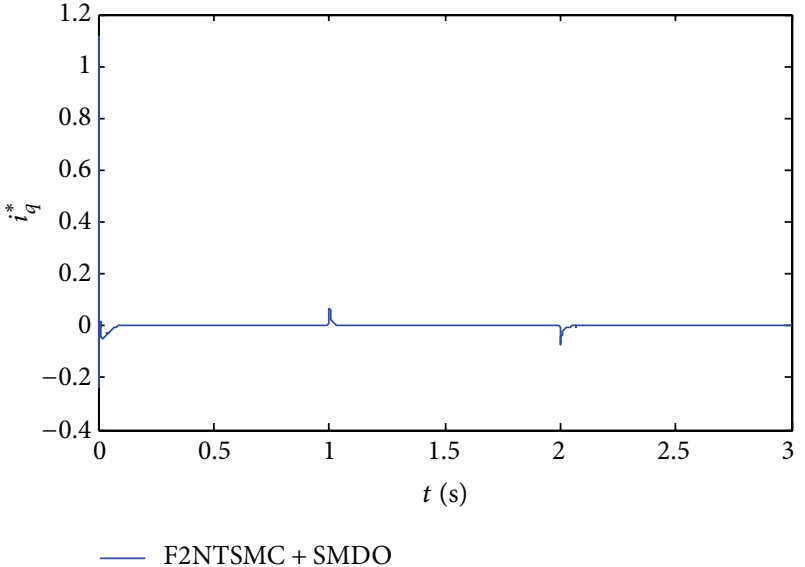

(b)

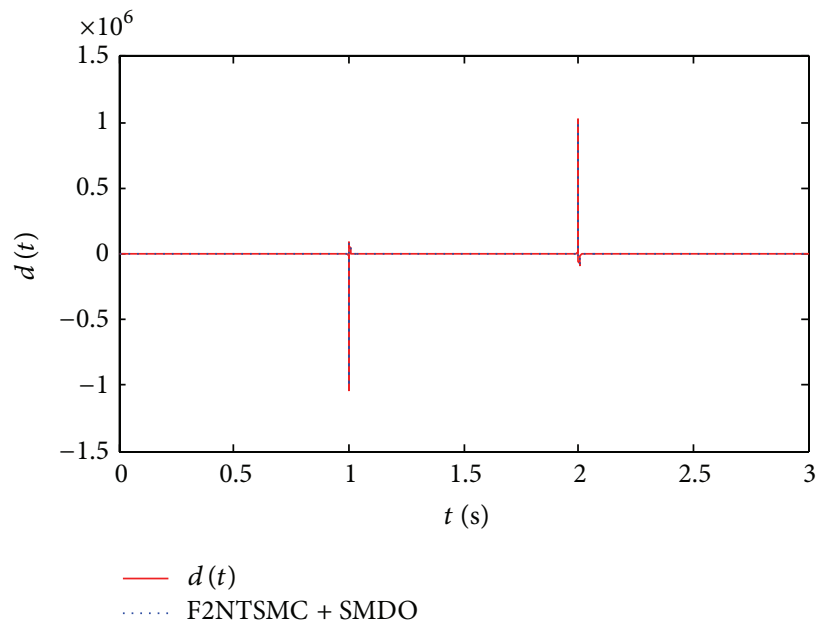

(c)

Figure 8: Robustness of SMDO-based F2NTSMC for the PMSM system with $d(t)$. (a) Position tracking performance, (b) current $i_{q}^{*}$, and (c) the lumped disturbances and their estimation.

Taking the time derivative on both sides of (53) and substituting (9), (10), and (55) into (50), the fractional-order sliding mode control input is obtained as

$$
\begin{aligned}
u_{\mathrm{FSMC}}= & -D^{1-r}\left(c_{3} \dot{e}+c_{4} z_{2}|e|^{y_{2}-1} \dot{e}+\varepsilon \operatorname{sgn}(s)\right) \\
& +\dddot{\theta}_{d}+\frac{k_{i}}{k_{p}} \ddot{\theta}^{(1-\lambda)}
\end{aligned}
$$

where $r=0.23, c_{3}=70.57, c_{4}=0.11, y_{2}=9.49$, and $\varepsilon=$ 76.03 .

The simulation results are presented in Figure 4 and demonstrate that the FTSMC has better performance of faster and higher tracking precision. However, the chattering phenomenon remains during the FTSMC process as shown in Figure 4(d). The position tracking performance of FTSMC and ITSMC is given in Figure 4(a). It can be seen from Figures 4(b) and 4(c) that tracking errors of FTSMC reach zero within finite time, but the convergence rate of ITSMC is far too slow. Furthermore, less chattering occurs during the FTSMC process than ITSMC according to Figures 4(d) and 4(e).

5.3. Simulation of I2NTSMC and F2NTSMC. In order to further accelerate the convergence rate and eliminate chattering effect, we adopt F2NTSMC combining 2NTSMC with FSMC. The control scheme takes advantages of less chattering of 2NTSMC and faster convergence rate, more precise tracking of FSMC.

The optimum parameters of F2NTSMC without $d(t)$ in (22) are $p=5, q=3, g=7, h=3, m=1, n=3, r=0.069$, $b_{1}=0.88, b_{2}=3.19, z=2.57, \alpha=4.38, \beta=0.0017, \phi=5.04$, and $\gamma=3.60$, respectively.

It is noticed from Figures 5(d) and 5(e) that there is neither singularity nor chattering during the F2NTSMC process. The actual position tracks the desired reference value more quickly than in the cases of I2NTSMC as seen 
in Figures 5(a), 5(b), and 5(c). To sum up, the F2NTSMC is better than the 2NTSMC.

\subsection{Simulation of SMDO-Based F2NTSMC}

5.4.1. SMDO-Based F2NTSMC for the Model with $d(t)$. The block diagram of the proposed SMDO-based F2NTSMC method is shown in Figure 6.

The position tracking control of the PMSM system with parameter uncertainties and external disturbances is considered. The optimal parameters of the F2NTSMC in (37) are calculated as $r=0.30, b_{1}=0.81, b_{2}=1.61, z=6.16$, $\alpha=1.04, \beta=0.0039, \phi=10.92$, and $\gamma=2.73$ and other parameters are as same as ones of F2NTSMC without $d(t)$. The optimal parameters of SMDO are given by $k_{o_{1}}=1257.21$ and $k_{o_{2}}=819.61$.

Simulation results are illustrated in Figure 7. The SMDObased F2NTSMC shows excellent tracking performance depicted in Figure 7(a). The position errors and sliding surface $s$ converge to the equilibrium points in finite time in spite of lumped disturbances including parameter uncertainties and external disturbances as seen from Figures 7(b) and 7(c), respectively.

The tracking performance of the F2NTSMC without SMDO is deteriorated due to too big lumped disturbances. Therefore, SMDO is applied to realize estimation and compensation in order to improve control performance and increase robustness of the system. From Figure 7(f), we can see that lumped disturbances can be effectively estimated. Moreover, Figures 7(d) and 7(e) demonstrate that the chattering phenomenon becomes effectively weakened.

5.4.2. Robustness of SMDO-Based F2NTSMC. In order to verify the robustness of $\mathrm{SMDO}$, the disturbance simulation is carried out. Figure 8 shows the system response as the load disturbances vary from $0 \mathrm{~N} \cdot \mathrm{m}$ to $2.5 \mathrm{~N} \cdot \mathrm{m}$ during the time range $[1,1.001] \mathrm{sec}$ and from $0 \mathrm{~N} \cdot \mathrm{m}$ to $-2.5 \mathrm{~N} \cdot \mathrm{m}$ during the time range $[2,2.001] \mathrm{sec}$.

The parameters of SMDO-based F2NTSMC are set as same as ones in Section 5.4.1. Figures 8(a) and 8(b) show the stability and robustness of SMDO-based F2NTSMC method. Figure $8(\mathrm{c})$ demonstrates that the SMDO can primely track the lumped disturbances.

\section{Conclusion}

In this paper, a SMDO-based F2NTSMC with strong robustness is developed to solve the position tracking control problem for the PMSM system in spite of parameter uncertainties and external disturbances. Simulation results show that the closed-loop system under the proposed F2NTSMC method has achieved fast convergence and high tracking precision. To further improve the disturbance rejection ability, SMDO is introduced to estimate and make compensation for the lumped disturbances. The combination of F2NTSMC and SMDO can obtain strong robustness and good dynamic performance. Simulation results have demonstrated the effectiveness and superiority of the proposed method.

\section{Conflict of Interests}

The authors declare that there is no conflict of interests regarding the publication of this paper.

\section{Acknowledgment}

This work was supported by the National Natural Science Foundation of China (Grant no. 61374147).

\section{References}

[1] Y. Feng, X. Yu, and F. Han, "High-order terminal slidingmode observer for parameter estimation of a permanentmagnet synchronous motor," IEEE Transactions on Industrial Electronics, vol. 60, no. 10, pp. 4272-4280, 2013.

[2] H. Liu and S. Li, "Speed control for PMSM servo system using predictive functional control and extended state observer," IEEE Transactions on Industrial Electronics, vol. 59, no. 2, pp. 11711183, 2012.

[3] L. Qi and H. Shi, "Adaptive position tracking control of permanent magnet synchronous motor based on RBF fast terminal sliding mode control," Neurocomputing, vol. 115, pp. 23-30, 2013.

[4] A. Khan, M. Uddin, and A. Rahman, "A novel wavelet neural network based robust controller for IPM motor drives," IEEE Transactions on Industry Applications, vol. 49, no. 5, pp. 23412351, 2013.

[5] A. Wang, X. Jia, and S. Dong, "A new exponential reaching law of sliding mode control to improve performance of permanent magnet synchronous motor," IEEE Transactions on Magnetics, vol. 49, no. 5, pp. 2409-2412, 2013.

[6] G. Maeng and H. H. Choi, "Adaptive sliding mode control of a chaotic nonsmooth-air-gap permanent magnet synchronous motor with uncertainties," Nonlinear Dynamics, vol. 74, no. 3, pp. 571-580, 2013.

[7] D. H. Ha, C. S. Lim, and D. S. Hyu, "Robust optimal nonlinear control with observer for position tracking of permanent magnet synchronous motors," Journal of Power Electronics, vol. 13, no. 6, pp. 975-984, 2013.

[8] M. Morawiec, "The adaptive backstepping control of permanent magnet synchronous motor supplied by current source inverter," IEEE Transactions on Industrial Informatics, vol. 9, no. 2, pp. 1047-1055, 2013.

[9] S. Chai, L. Wang, and E. Rogers, "Model predictive control of a permanent magnet synchronous motor with experimental validation," Control Engineering Practice, vol. 21, no. 11, pp. 15841593, 2013.

[10] D. Q. Dang, N. T. T. Vu, H. H. Choi et al., "Neuro-fuzzy control of interior permanent magnet synchronous motors: stability analysis and implementation," Journal of Electrical Engineering and Technology, vol. 8, no. 6, pp. 1439-1450, 2013.

[11] C.-H. Lin, "Recurrent modified Elman neural network control of PM synchronous generator system using wind turbine emulator of PM synchronous servo motor drive," International Journal of Electrical Power and Energy Systems, vol. 52, no. 1, pp. 143-160, 2013.

[12] S. Li, H. Liu, and S. Ding, "A speed control for a PMSM using finite-time feedback control and disturbance compensation," Transactions of the Institute of Measurement and Control, vol. 32 , no. 2, pp. 170-187, 2010. 
[13] W. Yu, Y. Luo, and Y. G. Pi, "Fractional order modeling and control for permanent magnet synchronous motor velocity servo system," Mechatronics, vol. 23, no. 7, pp. 813-820, 2013.

[14] B. T. Zhang and Y. G. Pi, "Enhanced robust fractional order proportional-plus-integral controller based on neural network for velocity control of permanent magnet synchronous motor," ISA Transactions, vol. 52, no. 4, pp. 510-516, 2013.

[15] C. B. Butt and M. A. Rahman, "Intelligent speed control of interior permanent magnet motor drives using a single untrained artificial neuron," IEEE Transactions on Industry Applications, vol. 49, no. 4, pp. 1836-1843, 2013.

[16] S. Li, M. Zhou, and X. Yu, "Design and implementation of terminal sliding mode control method for PMSM speed regulation system," IEEE Transactions on Industrial Informatics, vol. 9, no. 4, pp. 1879-1891, 2013.

[17] X. Yan, S. K. Spurgeon, and C. Edwards, "State and parameter estimation for nonlinear delay systems using sliding mode techniques," IEEE Transactions on Automatic Control, vol. 58, no. 4, pp. 1023-1029, 2013.

[18] X. Wei and L. Guo, "Composite disturbance-observer-based control and terminal sliding mode control for non-linear systems with disturbances," International Journal of Control, vol. 82, no. 6, pp. 1082-1098, 2009.

[19] S. Dadras and H. R. Momeni, "Fractional terminal sliding mode control design for a class of dynamical systems with uncertainty," Communications in Nonlinear Science and Numerical Simulation, vol. 17, no. 1, pp. 367-377, 2012.

[20] S. Zhankui and K. Sun, "Nonlinear and chaos control of a microelectro-mechanical system by using second-order fast terminal sliding mode control," Communications in Nonlinear Science and Numerical Simulation, vol. 18, no. 9, pp. 2540-2548, 2013.

[21] Y. Feng, X. Yu, and Z. Man, "Non-singular terminal sliding mode control of rigid manipulators," Automatica, vol. 38, no. 12, pp. 2159-2167, 2002.

[22] S. Yu, X. Yu, B. Shirinzadeh, and Z. Man, "Continuous finitetime control for robotic manipulators with terminal sliding mode," Automatica, vol. 41, no. 11, pp. 1957-1964, 2005.

[23] X. G. Yan, S. K. Spurgeon, and C. Edwards, "Sliding mode control for time-varying delayed systems based on a reducedorder observer," Automatica, vol. 46, no. 8, pp. 1354-1362, 2010.

[24] Y. Feng, X. Zheng, and X. Yu, "Second-order nonsingular terminal sliding mode decomposed control of uncertain multivariable systems," Asian Journal of Control, vol. 5, no. 4, pp. 505-512, 2003.

[25] M. P. Aghababa, "Design of a chatter-free terminal sliding mode controller for nonlinear fractional-order dynamical systems," International Journal of Control, vol. 86, no. 10, pp. 1744-1756, 2013.

[26] . Podlubny I, Fractional Differential Equations: An Introduction to Fractional Derivatives, Fractional Differential Equations, to Methods of Their Solution and Some of Their Applications, Academic press, Waltham, Mass, USA, 1998.

[27] J. Huang, H. Li, Y. Chen, and Q. Xu, "Robust position control of PMSM using fractional-order sliding Mode controller," Abstract and Applied Analysis, vol. 2012, Article ID 512703, 33 pages, 2012.

[28] S. Li, K. Zong, and H. Liu, "A composite speed controller based on a second-order model of permanent magnet synchronous motor system," Transactions of the Institute of Measurement and Control, vol. 33, no. 5, pp. 522-541, 2011.

[29] S. Li, K. Li, J. Wang et al., "Nonsingular and fast terminal sliding mode control method," Information and Control, vol. 38, no. 1, pp. 1-8, 2009.
[30] J. A. Moreno and M. Osorio, "A Lyapunov approach to secondorder sliding mode controllers and observers," in Proceedings of the 47th IEEE Conference on Decision and Control (CDC '08), pp. 2856-2861, IEEE, December 2008.

[31] M. Ö. Efe, "Fractional fuzzy adaptive sliding-mode control of a 2-DOF direct-drive robot arm," IEEE Transactions on Systems, Man, and Cybernetics B: Cybernetics, vol. 38, no. 6, pp. 15611570, 2008.

[32] S. P. Bhat and D. S. Bernstein, "Finite-time stability of continuous autonomous systems," SIAM Journal on Control and Optimization, vol. 38, no. 3, pp. 751-766, 2000. 


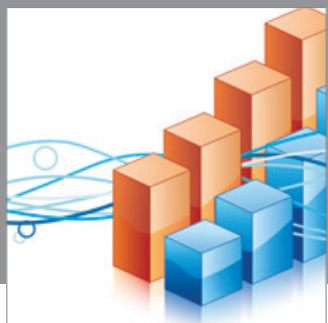

Advances in

Operations Research

mansans

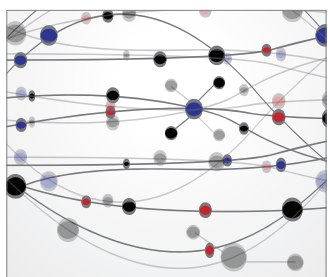

The Scientific World Journal
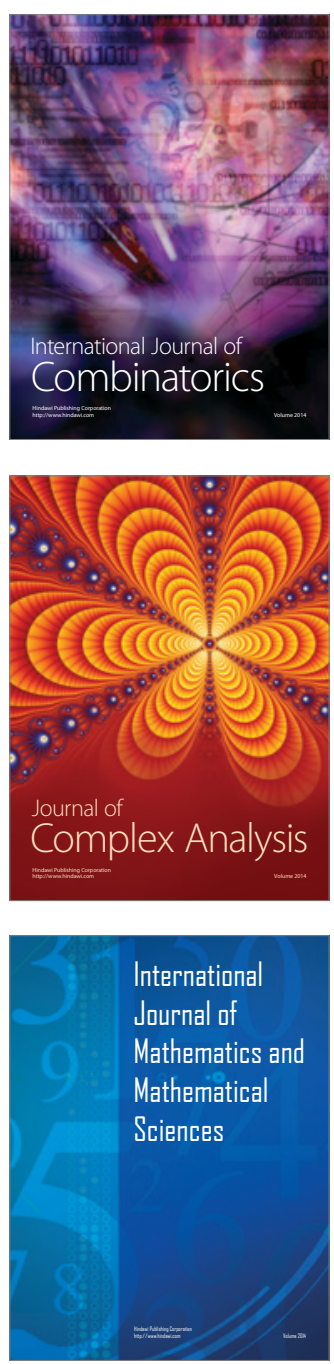
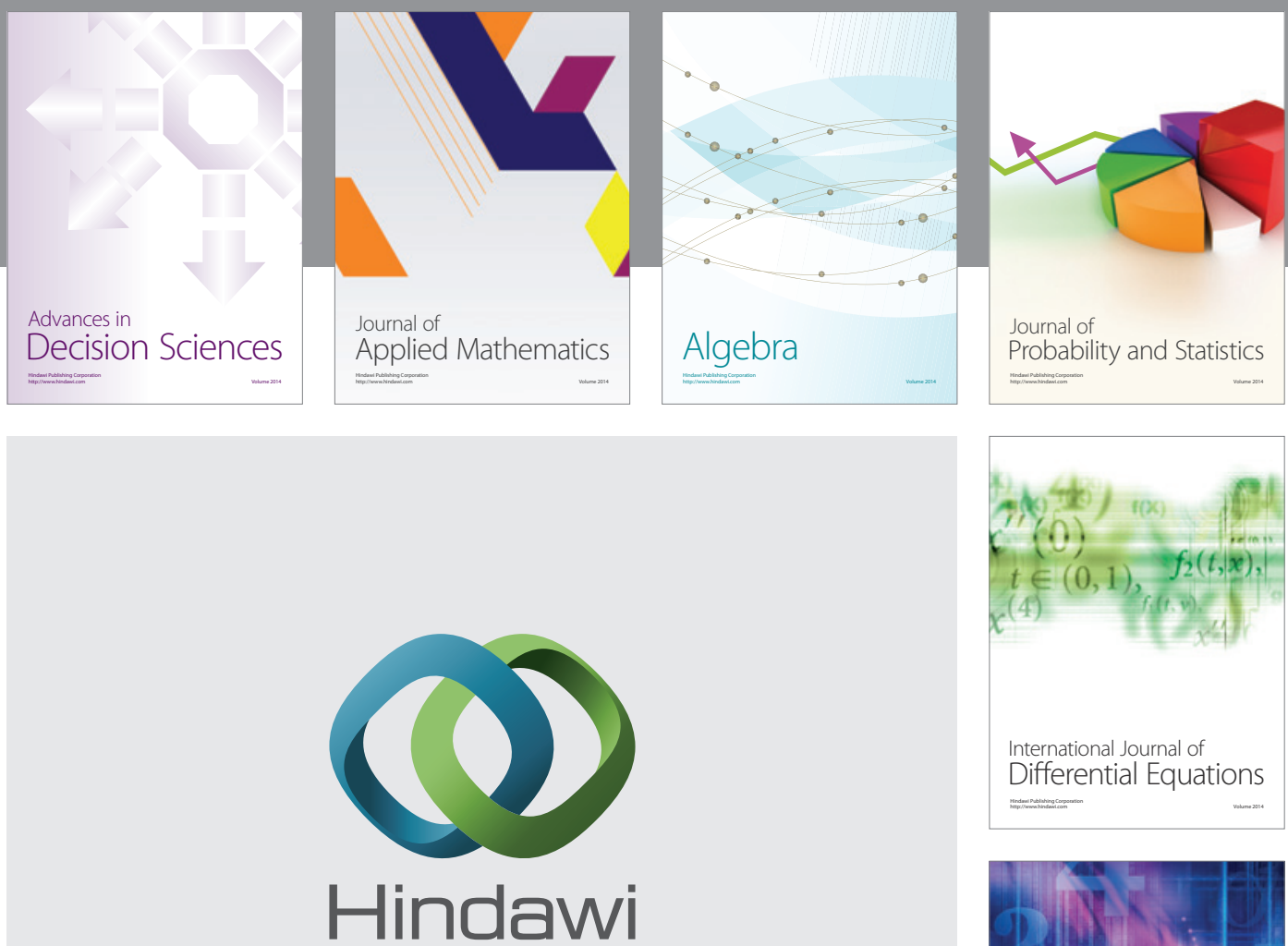

Submit your manuscripts at http://www.hindawi.com
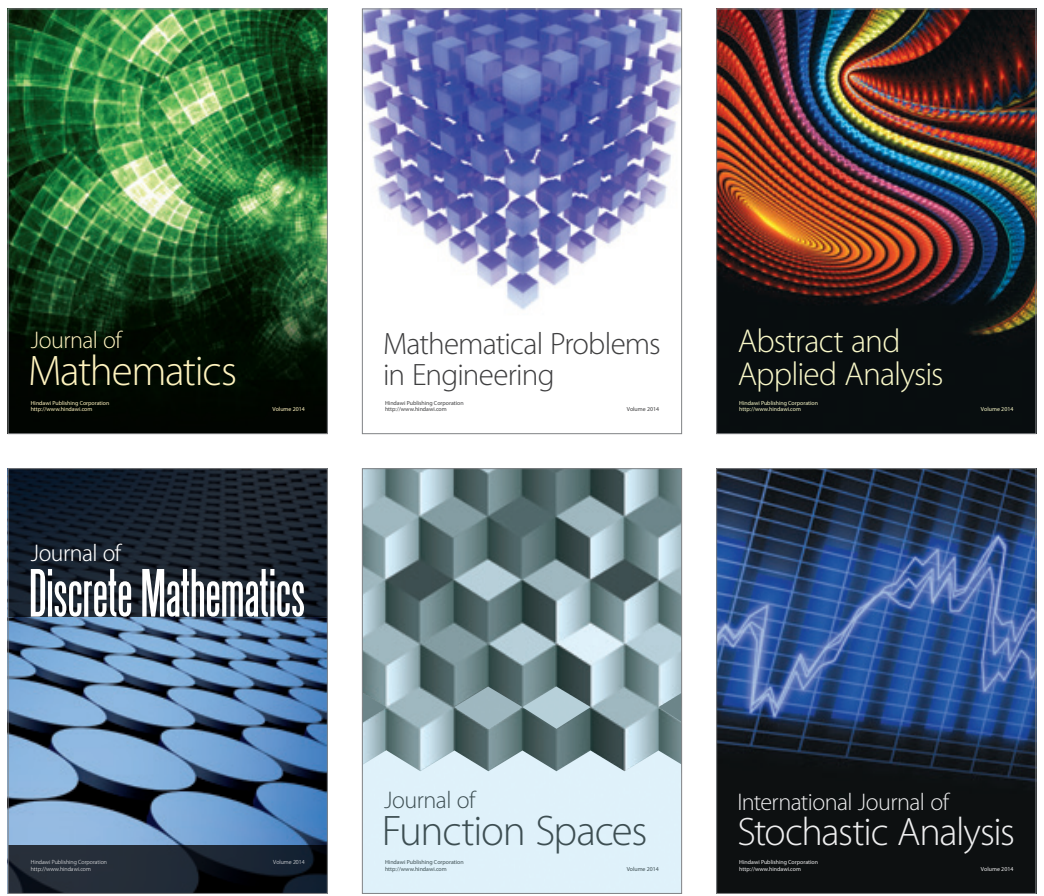

Journal of

Function Spaces

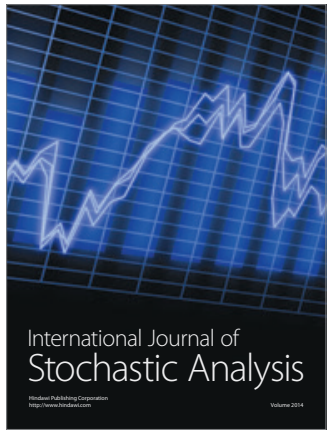

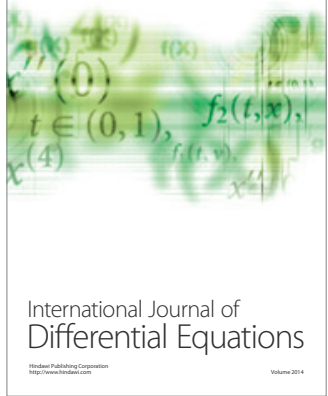
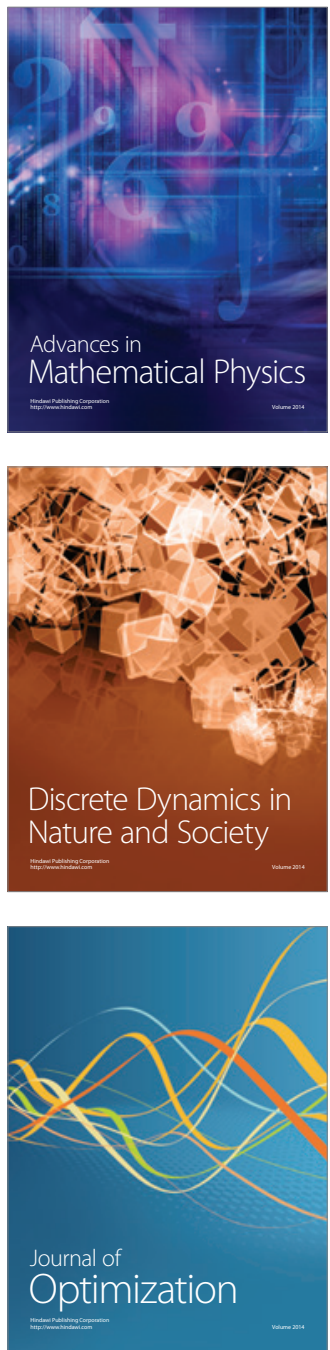